thus transforming the problem into one of a stationary, rather than a moving, medium. However, on applying the new boundary conditions, for $T^{*}$, there results another untabulated Laplace transform. In this case, the required transform may be found by a differentiation, rather than an integration, but the differentiation seems to involve a step of doubtful justifiability.

In conclusion, I wish to thank Dr. P. E. LeCorbeiller for his interest in this problem and for several helpful discussions of it.

\title{
ON A CLASS OF SINGULAR INTEGRAL EQUATIONS OCCURRING IN PHYSICS*
}

\author{
By H. P. THIELMAN (Iowa State College)
}

1. Introduction. Certain boundary value problems ${ }^{1}$ in electrodynamics can be formulated mathematically as Wiener-Hopf ${ }^{2}$ integral equations. The equations are of the form

$$
f(x)=\int_{0}^{\infty} K(|x-y|) g(y) d y, \quad x>0
$$

where $K(|x-y|)$ is a real function of the absolute value of the difference between $x$ and $y$. The Eq. (1) is an integral equation of the "first kind", $K(|x-y|)$ and $f(x)$ are given functions, and $g(y)$ is unknown. One advantage of formulating a given problem, if possible, as a Wiener-Hopf integral equation is that such equations are susceptible to the application of the theory of Fourier transforms. The application of the latter theory is especially advantageous when the Fourier transforms of the given functions are easily found. The object of the present paper is to show that for a certain class of Wiener-Hopf integral equations a more direct method yields the explicit solution in a more elementary and simpler way. The present method applies, however, only to those integral equations of type (1), and to integral equations of the second kind such as

$$
f(x)=g(x)-\lambda \int_{0}^{\infty} K(|x-y|) g(y) d y, \quad x>0
$$

if $K(|x-y|)$ satisfies the same linear homogeneous differential equations with constant coefficients in each of the regions $0 \leq y \leq x$, and $0 \leq x \leq y$. It should be mentioned here that the special case of Eq. (2) with $f(x) \equiv 0$, and $K(|x-y|)=e^{-|x-y|}$ was treated by Lalesco ${ }^{3}$ by a method similar to the one given here.

2. The general theory. We consider an integral equation of the form

$$
f(x)=\lambda \int_{0}^{\infty} K(|x-y|) g(y) d y \quad x>0
$$

*Received May 11, 1948.

1J. F. Carlson and A. E. Heins, The reflection of an electromagnetic plane wave by an infinite set of plates. I, Q. Appl. Math. 4, 313-329 (1947).

${ }^{2}$ N. Wiener, E. Hopf, Über eine Klasse singulärer Integralgleichungen, Sitzungsber. Preuss. Ak. Wissensch. 696-706 (1931).

${ }^{3}$ Trajan Lalesco, Théorie des équations intégrales, A. Hermann et Fils, Paris, 1912, p. 121. 
where

a) $\int_{0}^{\infty} K(|x-y|) d y$ is assumed to exist,

b) $K(|x-y|)$ is continuous for all positive $x$ and $y$,

c) $K(|x-y|)$ as a function of $x$ satisfies the same linear homogeneous differential equation with constant coefficients in each of the regions $0 \leq y \leq x$, and $0 \leq$ $x \leq y$.

Since $K(|x-y|)$ is a function of the absolute value of the difference $x-y$ only, we can write

$$
K(|x-y|)= \begin{cases}F(x-y) & \text { if } 0 \leq y \leq x, \\ F(-x+y) & \text { if } 0 \leq x \leq y\end{cases}
$$

By hypothesis $F(x-y)$, and $F(-x+y)$ as functions of $x$ satisfy the same linear homogeneous differential equation with constant coefficients. Let this be the differential equation of lowest order which they satisfy. It must then be of the form

$$
\left(\sum_{i=0}^{l} a_{2 i} D^{2 i}\right) u=0,
$$

where $2 l=n$, the order of the differential equation. This follows from the fact that for any $m$ which is a root of the characteristic equation of the linear differential equation satisfied by both $F(x-y)$ and $F(-x+y)$, the negative of $m$ is also a root. Let us write $\left(1^{\prime}\right)$ in the form

$$
f(x)=\lambda \int_{0}^{x} F(x-y) g(y) d y+\lambda \int_{x}^{\infty} F(-x+y) g(y) d y .
$$

Taking derivatives of this function and indicating by $F^{\prime}(x-y)$ the derivative with respect to the argument $|x-y|$,

$$
\frac{d}{d x} F(x-y)=F^{\prime}(x-y), \quad \frac{d}{d x} F(-x+y)=-F^{\prime}(-x+y),
$$

we obtain

$$
\begin{aligned}
& f^{\prime}(x)=\lambda \int_{0}^{x} F^{\prime}(x-y) g(y) d y-\lambda \int_{x}^{\infty} F^{\prime}(-x+y) g(y) d y \\
& f^{\prime \prime}(x)=\lambda \int_{0}^{x} F^{\prime \prime}(x-y) g(y) d y+2 \lambda F^{\prime}(0) g(x)+\lambda \int_{x}^{\infty} F^{\prime \prime}(-x+y) g(y) d y \\
& f^{\prime \prime \prime}(x)=\lambda \int_{0}^{x} F^{\prime \prime \prime}(x-y) g(y) d y+2 \lambda F^{\prime}(0) g^{\prime}(x)-\lambda \int_{x}^{\infty} F^{\prime \prime}(-x+y) g(y) d y .
\end{aligned}
$$

By mathematical induction it is easily shown that

$$
\begin{aligned}
f^{(2 i)}(x)=\lambda \int_{0}^{x} F^{(2 i)}(x-y) g(y) d y+\lambda \int_{x}^{\infty} F^{(2 i)}(-x+y) g(y) d y \\
\\
\quad+2 \lambda\left[F^{(2 i-1)}(0) g(x)+F^{(2 i-2)}(0) g^{\prime \prime}(x)+\cdots+F^{\prime}(0) g^{(2 i-2)}(x)\right],
\end{aligned}
$$

where $i=1,2, \cdots, l$. 
Forming the sum

$$
\sum_{i=0}^{l} a_{2 i} f^{(2 i)}(x)
$$

we obtain

$$
\sum_{i=0}^{l} a_{2 i} f^{(2 i)}(x)=2 \lambda \sum_{i=0}^{l-1} \sum_{i=t+1}^{l} a_{2 i} F^{(2 i-2 t-1)}(0) g^{(2 t)}(x),
$$

and the integrals have all cancelled each other because $F(x-y)$ and $F(-x+y)$ satisfy Eq. (A). We have thus found that $g(x)$ must satisfy a linear differential equation with constant coefficients of order $n-2$. Hence if $n=2, g(x)$ is explicitly expressed in terms of $f(x)$ and its second derivative. Equation $\left(2^{\prime}\right)$ gives a necessary condition to be a solution of $\left(1^{\prime}\right)$. In order to show that it is actually a soiution of $\left(1^{\prime}\right)$ we substitute the general solution of $\left(2^{\prime}\right)$ in $\left(1^{\prime}\right)$ and find the conditions on $f(x)$ in order that $g(x)$ may exist, and that the integral in $\left(1^{\prime}\right)$ may exist.

It is obvious that the theory just discussed applies not only to equations of the type $\left(1^{\prime}\right)$ but also to the homogeneous equation obtained from $\left(1^{\prime}\right)$ by letting $f(x)=g(x)$, the unknown function. If instead of starting with $\left(1^{\prime}\right)$ we start with the integral equation of the "second kind" (2), where $K(|x-y|)$ is of the type specified above, we obtain in an analogous manner the conditions on $g(x)$ that it may be a solution of (2).

It should be noticed that the kernels of the form

$$
K(|x-y|)=\sum_{i=1}^{m} e^{-k_{i}|x-\nu|} P_{i}(|x-y|),
$$

where $k_{i}>0$ and $P_{i}(|x-y|)$ are polynomials in $|x-y|$, are of the required type.

We shall illustrate this general theory by some examples which in themselves are of sufficient generality to warrant an independent investigation. For this reason the results obtained will be stated as theorems. The derivations of these results will illustrate the applicability of the method to problems in applied mathematics in which the reader may be interested.

3. Application of the general theory. By an application of the above theory we prove the

Theorem I. Necessary and sufficient conditions that $g(x)$ be a solution of the integral equation

$$
f(x)=\lambda \int e^{-k|x-y|} g(y) d y \quad x>0, k>0
$$

are

a) that $k f(0)-f^{\prime \prime}(0)=0$,

b) $g(x)=\frac{k^{2} f(x)-f^{\prime \prime}(x)}{2 k \lambda}$

c) that the integral in (B) exist, namely that $g(x)$ be of the order $e^{e x}$ where $c<k$.

Proof. We note that the kernel satisfies the differential equation $\left(k^{2}-D^{2}\right) u=0$. Writing (B) in the form

$$
f(x)=\lambda \int_{0}^{x} e^{-k(x-y)} g(y) d y+\lambda \int_{x}^{\infty} e^{-k(y-x)} g(y) d y
$$


and differentiating with respect to $x$ twice we obtain

$$
\begin{aligned}
& f^{\prime}(x)=-\lambda k \int_{0}^{x} e^{-k(x-y)} g(y) d y+\lambda g(x)+\lambda k \int_{x}^{\infty} e^{-k(y-x)} g(y) d y-\lambda g(x), \\
& f^{\prime \prime}(x)=\lambda k^{2} \int_{0}^{x} e^{-k(x-y)} g(y) d y-2 \lambda g(x)+\lambda k^{2} \int_{x}^{\infty} e^{-k(y-x)} g(y) d y .
\end{aligned}
$$

Forming $k^{2} f(x)-f^{\prime \prime}(x)$ we get $k^{2} f(x)-f^{\prime \prime}(x)=2 \lambda k g(x)$, or

$$
g(x)=\frac{k^{2} f(x)-f^{\prime \prime}(x)}{2 \lambda k} .
$$

Substituting this value with $x$ replaced by $y$ into $\left(\mathrm{B}^{\prime}\right)$ there results

$$
\begin{aligned}
f(x)= & \frac{1}{2 k} \int_{0}^{x} e^{-k(x-y)}\left[k^{2} f(y)-f^{\prime \prime}(y)\right] d y \\
& \quad+\frac{1}{2 k} \int_{x}^{\infty} e^{-k(y-x)}\left[k^{2} f(y)-f^{\prime \prime}(y)\right] d y \\
= & \frac{k}{2} \int_{0}^{x} e^{-k(x-y)} f(y) d y+\frac{k}{2} \int_{x}^{\infty} e^{-k(y-x)} f(y) d y \\
& \quad-\frac{1}{2 k} \int_{0}^{x} e^{-k(x-y)} f^{\prime \prime}(y) d y-\frac{1}{2 k} \int_{x}^{\infty} e^{-k(y-x)} f^{\prime \prime}(y) d y .
\end{aligned}
$$

Integrating the integrals involving $f^{\prime \prime}(y)$ by parts twice by letting first $d v=f^{\prime \prime}(y) d y$, and then $d v=f^{\prime}(y) d y$ we get

$$
f(x)=f(x)-\frac{e^{k x}}{2 k}\left[k f(0)-f^{\prime}(0)\right] .
$$

If this last equation is to be an identity $k f(0)-f^{\prime}(0)=0$, as stated in the theorem. The function $g(x)$ has to be of the order $O\left(e^{c x}\right)$ in order for the integral to exist.

As a direct consequence of Theorem I we have

Theorem II. All twice differentiable solutions of the homogeneous integral equation

$$
g(x)=\lambda \int_{0}^{\infty} e^{-k|x-y|} g(y) d y
$$

are

a) $\quad g(x)=c_{1}\left(k^{1 / 2} \sin \left\{[k(2 \lambda-k)]^{1 / 2} x\right\}+(2 \lambda-k)^{1 / 2} \cos \left\{[k(2 \lambda-k)]^{1 / 2} x\right\}\right)$

$$
\text { if } \lambda>\frac{k}{2}
$$

b) $g(x)=c_{2}(1+k x) \quad$ if $\quad \lambda=\frac{k}{2}$,

c) $\quad g(x)=c_{3}\left(k^{1 / 2} \sinh \left[k(k-2 \lambda)^{1 / 2}\right] x+(k-2 \lambda)^{1 / 2} \cosh \left[k(k-2 \lambda)^{1 / 2}\right] x\right)$

if $0<\lambda<k / 2$, where the $c_{i}(i=1,2,3)$ are arbitrary constants. 
Proof. By setting $f(x)$ in Theorem I equal to $g(x)$ we obtain $k g(0)-g^{\prime}(0)=0$, $g(x)=k^{2} g(x)-g^{\prime \prime}(x) / 2 \lambda k$. Rewriting the last equation for $g(x)$ we obtain

$$
g^{\prime \prime}(x)+k(2 \lambda-k) g(x)=0 .
$$

We have now three possible cases: a) $2 \lambda-k>0$, b) $2 \lambda-k=0$, and c) $2 \lambda-k<0$.

For case a) the general solution of the differential equation satisfied by $g(x)$ is

$$
g(x)=A \sin [k(2 \lambda-k)]^{1 / 2} x+B \cos [k(2 \lambda-k)]^{1 / 2} x .
$$

Imposing the condition a) of Theorem I on $g(x)$, i.e. $k g(0)-g^{\prime}(0)=0$, we find that $B / A=[(2 \lambda-k) / k]^{1 / 2}$, or

$$
g(x)=c_{1}\left(k^{1 / 2} \sin [k(2 \lambda-k)]^{1 / 2} x+(2 \lambda-k)^{1 / 2} \cos [k(2 \lambda-k)]^{1 / 2} x\right) .
$$

For case b), when $2 \lambda=k, g^{\prime \prime}(x)=0$, and $g(x)=c_{1}(1+k x)$ where $c_{2}$ is an arbitrary constant.

For case c) $2 \dot{\lambda}-k<0$, and

$$
g(x)=A \sinh [k(k-2 \lambda)]^{1 / 2} x+B \cosh [k(k-2 \lambda)]^{1 / 2} x .
$$

Since, however, $k g(0)-g^{\prime}(0)=0$,

$$
g(x)=c_{3}\left(k^{1 / 2} \sinh [k(k-2 \lambda)]^{1 / 2} x+(k-2 \lambda)^{1 / 2} \cosh [k(k-2 \lambda)]^{1 / 2} x\right) .
$$

$g(x)$ has to be of order $O\left(e^{c x}\right)$ with $c<k$, hence $[k(k-2 \lambda)]^{1 / 2}<k$ or $\lambda>0$.

We see that the characteristic values, $\lambda$, constitute a continuous spectrum, a phenomenon which is due entirely to the fact that our integral equation has an unbounded region of integration. For integral equations with bounded kernels, and bounded regions of integration it is well known that the number of characteristic values is at most denumerable. If in Theorem II we set $k=1$, we obtain the example treated by Lalesco ${ }^{3}$.

By direct application of the general theory we obtain Theorem III. If $g(x)$ is a solution of the integral equation

$$
f(x)=\lambda \int_{0}^{\infty} e^{-k|x-y|}|x-y| g(y) d y
$$

then $g(x)$ must satisfy the differential equation

$$
g^{\prime \prime}(x)+k^{2} g(x)=\frac{f^{i v}(x)-2 k^{2} f^{\prime \prime}(x)+k^{4} f(x)}{2 \lambda}
$$

Proof. The kernel satisfies the differential equation

$$
\left(D^{4}-2 k^{2} D+k^{4}\right) u(x)=0,
$$

in each of the regions $0 \leq y \leq x$, and $0 \leq x \leq y$. Writing $(D)$ in the form

$$
f(x)=\lambda \int_{0}^{x} e^{-k(x-y)}(x-y) g(y) d y+\lambda \int_{x}^{\infty} e^{-k(y-x)}(y-x) g(y) d y,
$$

differentiating four times and forming the expression on the left of the following equation we obtain,

$$
f^{i v}(x)-2 k^{2} f^{\prime \prime}(x)+k^{4} f(x)=2 \lambda k^{2} g(x)+2 \lambda g^{\prime \prime}(x),
$$


which can be written as

$$
g^{\prime \prime}(x)+k^{2} g(x)=\frac{f^{i v}(x)-2 k^{2} f^{\prime \prime}(x)+k^{4} f(x)}{2 \lambda} .
$$

If we set $f(x) \equiv g(x)$ in (D) we obtain the Theorem IV. If $g(x)$ is a solution of the homogeneous equation

$$
g(x)=\lambda \int_{0}^{\infty} e^{-k|x-y|}|x-y| g(y) d y \quad x>0, k>0
$$

then $g(x)$ must satisfy the differential equation

$$
g^{i v}(x)-2\left(\lambda+k^{2}\right) g^{\prime \prime}(x)-k^{2}\left(2 \lambda-k^{2}\right) g(x)=0 .
$$

The real parts of the roots of the characteristic equation of the last differential equation have to be in absolute value less than $k$ in order that the integral may exist with $g(x)$ a solution of $(\mathrm{E})$. The complete discussion of the spectrum of characteristic values of this equation, as well as the treatment of certain integral equations of the second kind is reserved for another paper.

\section{ON THE GENERAL EQUATIONS OF PROBLEMS OF AXIAL SYMMETRY IN THE THEORY OF PLASTICITY*}

\section{BY P. S. SYMONDS (Brown University)}

1. Introduction. The solutions of axially symmetric problems in plasticity which have been presented in the literature depend in general upon the assumption of the so-called "full plasticity" condition. According to this assumption, two principal stress differences are taken equal to the yield stress in pure tension. This implies that two of the principal stresses are equal, and thus reduces the problem to a statically determinate one. On this basis solutions have been given for a number of important problems, such as those of the indentation of a rigid surface of revolution into a plastic mass (see for example papers of Hencky ${ }^{1}$ and Ishlinsky ${ }^{2}$ ).

This is a quite fictitious yield condition, with little physical or mathematical justification. Hence it is of interest to examine the general equations for axial symmetry using the well confirmed Mises flow condition, and, in particular, to determine whether or not these equations form a system of hyperbolic type. If so, the techniques of numerical integration using the networks of characteristic curves, which have been developed for supersonic fluid flow problems, could be applied to these problems of plasticity. Unfortunately the answer to this question, as shown in the present note, is that the equations are actually of elliptic type, the "characteristics" being real only under the special condition which reduces the problem to one of plane strain.

\footnotetext{
*Received July 8, 1948. The results presented here were obtained in the course of research conducted under a contract sponsored jointly by the Office of Naval Research and the Bureau of Ships.

${ }^{1} \mathrm{H}$. Hencky, Über einige statisch bestimmte Fälle des Gleichgewichts in plastischen Körpern, $\mathrm{Z}$. angew. Math. Mech. 3, 241-251 (1923).

${ }^{2} \mathrm{~A}$. Ishlinsky, The problem of plasticity with axial symmetry and Brinell's test, Prikl. Mat. Mekh. 8, 201-224 (1944).
} 